\title{
Group-Based Joint Coding and Embedding Technique for Multimedia Fingerprinting
}

\author{
Shan He and Min Wu \\ ECE Department, University of Maryland, College Park, U.S.A
}

\begin{abstract}
This paper proposes a group-based fingerprinting scheme employing a joint coding and embedding strategy to trace multimedia distribution and proactively prevent the leak of multimedia information. Taking advantage of the prior knowledge on the collusion pattern, we construct compact fingerprints that consist of user sub-codeword and group sub-codeword and are embedded in host signal via spread spectrum technique. The detection is done in two levels, which identifies guilty groups through correlation and then narrows down to specific colluders through minimum distance decoding. Experimental results show that the proposed method provides higher collusion resistance than the existing non-grouped fingerprint codes.
\end{abstract}

Keywords: Multimedia Fingerprinting, Collusion Resistance, Error Correcting Code, Group-Based Fingerprinting

\section{INTRODUCTION}

With the development of the Internet and multimedia processing techniques, the protection of multimedia content has become increasingly important. Digital fingerprinting is an emerging technology to protect multimedia from unauthorized redistribution [1]. It embeds a unique ID into each user's copy, which can be extracted to help identify culprits when an unauthorized leak is found. A powerful, cost-effective attack is the collusion attack from a group of users, where the users combine their copies of the same content but with different fingerprints to generate a new version. If designed improperly, the fingerprints can be attenuated or even removed by the collusion attack.

Several techniques have been proposed in the literature to provide collusion resistance in multimedia fingerprinting systems. One category is the non-coded fingerprinting. A typical example is the orthogonal fingerprinting, where each user is assigned a spread spectrum sequence as fingerprint and the sequence is mutually orthogonal to those for other users. The collusion resistance performance of orthogonal fingerprinting has been well studied in [2]. Later, Wang et al. [3] observed that some users may be more likely to collude with each other due to cultural and other relations. Incorporating this prior knowledge, they introduce correlation among users' fingerprints and build group-based fingerprints based on orthogonal fingerprinting. The group-based fingerprint can improve the collusion resistance of the orthogonal fingerprinting when the prior knowledge is accurate. A weakness of the orthogonal fingerprinting scheme is that the number of basis sequences needed and the complexity of detection would increase linearly with the number of users. The second category, known as coded fingerprinting, employs codes and coded modulation to build the fingerprint. Early works on coded fingerprinting focused on fingerprinting generic data. Boneh and Shaw introduced a two-level construction in code domain to resist up to $c$ colluders with high probability [4]. A number of follow-up works investigate fingerprinting codes for generic data with shorter length and faster decoding for digital fingerprinting [5] [6]. In the existing digital fingerprinting works, identifiable parent property (IPP) code and traceability (TA) code are widely studied [7] [8]. In [9], Safavi-Naini et al. applied $c$-TA codes, which can be constructed from error correcting code (ECC), to multimedia fingerprinting. We shall call it ECC-based Fingerprinting. Based on this fingerprinting scheme, Fernandez et al. utilized Guruswami-Sudan soft-decision list decoding algorithm to find all provably identifiable dishonest users [10]. Since most of the existing coded fingerprinting works focused on generic data, they did not explore the special properties of multimedia signal during fingerprint design. Although some papers [9] [10] claimed that their schemes are for multimedia, the fingerprint embedding was handled only through the marking

The authors can be reached at shanhe@glue.umd.edu and minwu@eng.umd.edu. 
assumption [4]. For example, it typically assumes that colluders can only change fingerprint codebits in which they have different values. The marking assumption works well for generic data but does not fully account for the multimedia. In our recent work, we replaced the marking assumption with real embedding of the ECC based fingerprint for multimedia applications and studied its overall performance under various cases [11]. The results showed that the code structure and the relative small alphabet size provide ECC based fingerprinting a compact representation of users with less basis sequences. These features also result in low complexity of detection but low collusion resistance .

In this paper, we focus on approaches to improve the collusion resistance of ECC based fingerprinting while retaining its ability of efficient detection. In contrast to many existing works that emphasize on the coding layer and often omit the embedding layer, we find it beneficial to jointly design the coding and embedding layers. We develop a new fingerprinting system dubbed as GRACE (Group-Based Joint Coding and Embedding) fingerprinting. GRACE fingerprinting takes advantage of the group structure appearing among users and jointly considers the coding and embedding of the information for both group and individual users. As shown in our analysis and experiments, the joint coding and embedding for grouping approach substantially improves the collusion resistance of the existing ECC based fingerprinting while preserving the compact representation of fingerprints and low complexity of detection.

This paper is organized as follows. Section 2 introduces the existing ECC based fingerprinting. We describe our proposed GRACE fingerprinting system in Section 3. Its effectiveness is demonstrated in Section 4 through experiments. Discussions and conclusions are presented in Section 5 and Section 6 respectively.

\section{ECC BASED FINGERPRINTING SYSTEMS}

A typical framework of ECC based multimedia fingerprinting [11] includes a code layer and a spread spectrum based embedding layer. For anti-collusion purpose, the fingerprint code is constructed as a $c$-TA code in [9] and each codeword is assigned to one user as the fingerprint. A $c$-TA code satisfies the condition that any colluded version of the codewords by any $c$ colluders have closer distance to at least one of these colluders' codewords than to the innocents'. We can construct a $c$-TA code using established ECC over an alphabet of size $q$, provided the minimum distance $D$ is large enough and satisfies

$$
D>\left(1-\frac{1}{c^{2}}\right) L
$$

Here $L$ is the code length and $c$ is the number of colluders that the code is intended to resist [9]. With the minimum distance achieving the Singleton bound, Reed-Solomon code is a natural choice in ECC based fingerprinting. The number of $c$-TA codewords over an alphabet of size $q$ using Reed-Solomon code is $N_{u}=q^{t}$, where $t=\left\lceil L / c^{2}\right\rceil$. To embed a codeword, we first partition the host signal into $L$ non-overlapped segments with one segment corresponding to one symbol. Then we build $q$ mutually orthogonal spread spectrum sequences with identical energy to represent the $q$ possible symbol values in the alphabet. One of the $q$ sequences is added into each segment (with perceptual scaling) according to the symbol value in the fingerprint codeword. The concatenation of all fingerprinted segments forms the ultimate fingerprinted signal.

After the distribution of the fingerprinted copies, users may collaborate and mount cost-effective collusion attacks. The existing works on coded fingerprinting have primarily targeted at code-level collusion resistance. Therefore the interleaving collusion is a widely considered collusion model. In interleaving collusion, each colluder contributes a non-overlapped set of segments (corresponding to symbols) and these segments are assembled to form a colluded copy. The other type of collusion is signal domain collusion attack. A typical example is the averaging collusion, whereby colluders average the corresponding components in their copies to generate a colluded version. In both types of collusion, the colluders generally make contributions of approximately equal amount to share the risk of being captured. Additional distortion may be added to the colluded signal, which is typically modelled as an additive noise.

A common goal considered in the fingerprinting literature is to catch one colluder with high probability. At the detector side, we first determine the symbol that is most likely to be present in each multimedia segment using a correlation detector commonly used for spread spectrum embedding [12]. We then search the codebook and identify the colluder to be the one whose codeword has the smallest Hamming distance to the extracted codeword. 


\section{THE PROPOSED GROUP-BASED JOINT CODING AND EMBEDDING FINGERPRINTING}

A very important observation is that a user is often not equally likely to collude with other users. For example, users in the same geographic area or having similar social or cultural background are more likely to collude [3]. Thus we can put users into several groups and add additional information to each user's copy to facilitate detection. On the other hand, the results in our previous work show that as the fingerprint energy increases, the collusion resistance of ECC fingerprinting only increases a little compared with the huge increment in the case of orthogonal fingerprinting [11]. Previous results suggest that as fingerprint energy increases, the symbol detection accuracy is high enough and the performance bottleneck lies in the outer code structure. This observation makes it feasible to use part of the fingerprint energy to embed additional information to facilitate the colluder detection while keeping the symbol detection accuracy high enough. To take advantage of these two observations, we propose the GRACE fingerprinting system. In the GRACE fingerprinting, based on the code construction we overlappingly embed the group information and the user information so that they are seamlessly combined and spread all over the host signal. As we shall see, this joint coding and embedding brings great advantages in terms of the collusion resistance and the security of the fingerprinting scheme.

\subsection{Fingerprint Construction}

Based on the prior knowledge on the collusion pattern, we put the fingerprint codewords into groups and then add symbols representing the group information. We call these symbols "group sub-codeword", and refer to the symbols for distinguishing individual users as "user sub-codeword".

To construct the user sub-codewords, we start with a fingerprint codebook $C$ based on error correcting code construction over an alphabet of size $q$, such as those in [9] [11]. The code length is $L$, and the minimum distance is $D$ which is typically less than $L$. We then rearrange the codebook into groups so that within each group the codewords are orthogonal to each other, i.e. users within the group have distinct values at each symbol position. Thus the code distance within a group equals the codeword length $L$. We assign each codeword to a user as the user sub-codeword. This process is illustrated in Fig. 1 and described in more detail below.

1. Set the group index $i=1$, initialize the set of codewords for group $i$ to be empty, $G(i)=\varnothing$;

2. Pick any codeword $c \in C$ to be the first element for group $i$, move it from $C$ to group $i: G(i)=\{G(i), c\}$, $C \leftarrow C-\{c\}$.

3. Examine every codeword in $C$ : If $c \in C$ is orthogonal to all the existing codewords in $G(i)$, move $c$ from $C$ to $G(i)$.

4. If $C \neq \varnothing$, continue to build the next group. That is $i \leftarrow i+1$, initialize $G(i)=\varnothing$, and go back to step 2 .

The group sub-codewords are designed to be orthogonal to each other. One simple way to construct the group sub-code is to use one symbol to represent one group, thus totally we need $g$ symbols for $g$ groups. For each group, we construct repetition code with length $L$ by repeating the symbol $L$ times as the group sub-codeword.

\subsection{Fingerprint Embedding}

In the proposed GRACE fingerprinting scheme, we embed both the group sub-codeword and user sub-codeword by mapping them to spreading sequences and then combining them overlappingly to add to host signal.

In the signal domain, to embed the fingerprint codeword into host signal, we partition the host signal into $L$ segments, where each segment corresponds to one symbol, and $L$ is the user sub-codeword length. Then for each symbol in a user sub-codeword or a group sub-codeword, we generate a spreading sequence that will be added into one segment of the host signal. The group information of the GRACE is orthogonal to and overlaps the spreading sequence conveying the user sub-codeword [13]. More specifically, we use the sequences $\left\{\mathbf{u}_{j}, j=1 \ldots q\right\}$ to represent $q$ symbol values in the alphabet of user sub-codeword, where $\mathbf{u}_{j}$ 's are orthogonal to each other and have identical energy $\|\mathbf{u}\|^{2}$. The $g$ sequences $\left\{\mathbf{a}_{i}, i=1 \ldots g\right\}$ represent $g$ groups. They are orthogonal to each other and to $\left\{\mathbf{u}_{j}\right\}$, and have the same energy as $\mathbf{u}_{j}$ 's, i.e. $\|\mathbf{a}\|^{2}=\|\mathbf{u}\|^{2}$. We then construct the fingerprint sequence for the $j^{\text {th }}$ segment of user $k$ who belongs to group $i$ as

$$
\mathbf{s}_{i j k}=\sqrt{1-\rho} \mathbf{u}_{s y m(k, j)}+\sqrt{\rho} \mathbf{a}_{i}
$$




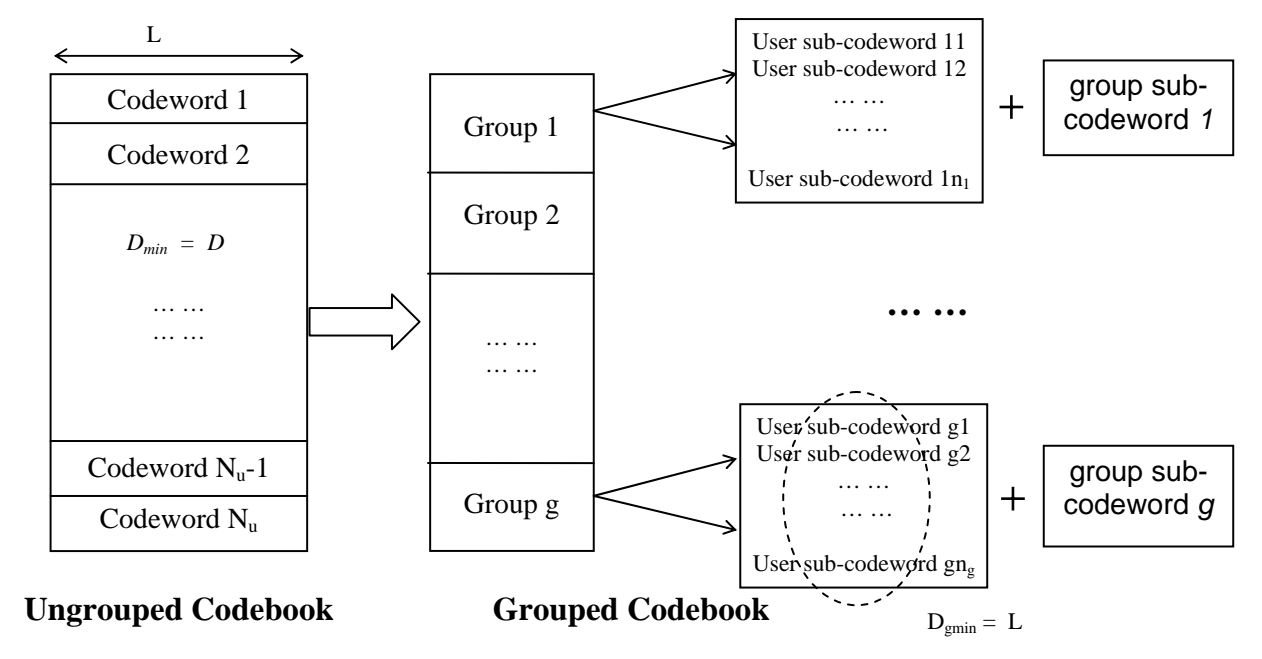

Figure 1. Fingerprint codeword construction for GRACE fingerprinting

where function $\operatorname{sym}(k, j)$ is used to retrieve the symbol in $j^{\text {th }}$ coordinate of user $k$ 's user sub-codeword, and $\rho$ is used to adjust the relative energy between the group sub-codeword and user sub-codeword. This fingerprint signal is finally added to the $j^{\text {th }}$ segment of the host signal. A higher $\rho$ puts more energy on group information and thus provides a more accurate detection of the guilty groups. However, this also reduces the accuracy of user sub-codeword detection, which may lead to low accuracy of colluder detection. Therefore, there is a trade-off between group detection and user detection when choosing $\rho$. Since in our scheme we have $L$ segments to collect the energy for group detection and usually collusion happens among a small number of groups, we can choose a small $\rho$ to guarantee the user sub-codeword detection with an acceptable group detection accuracy.

We can see that one of the important step in the GRACE fingerprinting is the joint coding and embedding, where we embed the spreading sequence of group sub-codeword overlappingly with that of the user sub-codeword. Alternatively, we can embed the group information by appending the spreading sequence of group sub-codeword to that of user sub-codeword. This appending scheme is equivalent to the appending of the group sub-codeword in the code level without taking advantage of embedding. To demonstrate the performance gain of the GRACE fingerprinting brought by the joint consideration of coding and embedding, we shall present this appending scheme as well and refer to it as the appending group ECC fingerprinting. In this fingerprinting scheme, the equivalent codeword for each user is the concatenation of the user sub-codeword with length $L$ and the group sub-codeword with length $L_{g}$, where $L_{g}$ is not necessary equal to $L$. Thus the total codeword length is $L+L_{g}$. To embed this codeword, the host signal is partitioned into $L+L_{g}$ segments. The corresponding spread sequence is added into each segment according to the codeword symbols. For a given host signal, where the total number of embeddable signal samples $N$ is fixed, the longer the group sub-codeword, the smaller each segment length $N_{s}^{\prime}=N /\left(L+L_{g}\right)$. Thus $L_{g}$ is used to adjust the relative energy between the group sub-codeword and the user sub-codeword.

\subsection{Fingerprint Detection}

At the detector side, we have a two-level scheme. First, we examine through a correlation detector the group information in the colluded signal to identify the groups from which the colluders are from. We then focus our attention on these identified guilty groups and apply ECC decoding [11] to narrow down the colluders. More specifically, we extract group information from the test signal $\mathbf{z}$ using a non-blind correlation detector, the detection statistic with respect to group $i$ is

$$
T_{G}(i)=\frac{(\mathbf{z}-\mathbf{x})^{T} \mathbf{b}_{i}}{\|\mathbf{b}\|}, \quad i=1,2, \ldots, g
$$


where $\mathbf{x}$ is the host signal which is often available in the fingerprinting application, and $\mathbf{b}_{i}$ is the concatenation of the sequences representing group $i$ 's information from each segment. In the above settings, $\mathbf{b}_{i}=\left[\mathbf{a}_{i}^{T} \ldots \mathbf{a}_{i}^{T}\right]^{T}$ since we embed $\mathbf{a}_{i}$ in each segment of group $i$. The $k^{\text {th }}$ group is considered guilty for the test signal if $T_{G}(k)>h$, where $h$ is the threshold. The union of the detected guilty groups forms the guilty group set.

To narrow down the colluders inside the guilty groups, we first extract the (colluded) user sub-codeword symbols from each segment of the test signal $\mathbf{z}$ through the maximum correlation detector with each of the $q$ spreading sequences $\mathbf{u}_{j}$ [11]. We then examine the codebook and apply the decoding algorithm used in most ECC based fingerprinting systems [9] [11] to find the closest codeword to the extracted codeword within the guilty groups. The detector declares the user corresponding to this codeword as the colluder.

The detection for the appending group ECC fingerprinting is similar to GRACE fingerprinting. We first extract the group information and user codeword from corresponding segments through a non-blind correlation detector. The identification of colluder is then performed within the guilty groups.

\section{EXPERIMENTAL RESULTS}

In this section, we demonstrate the effectiveness of the proposed GRACE fingerprinting through experiments on synthesis signal and real images.

\subsection{Simulations on Synthetic Signals}

To build the user sub-codewords, we employ a Reed-Solomon code with $q=32, L=30, N_{u}=1024, D=29$ and rearrange it into 32 groups using the algorithm described in section 3.1. Inside each group there are 32 codewords mutually orthogonal to each other. We choose $\rho=1 / 7$ in Eqn.(2) to generate the fingerprint signal from the user sub-codeword and the group sub-codeword in GRACE. For fair comparison, we choose $L_{g}=5$ for appending group ECC fingerprinting. We use the repetition codewords described in section 3.1 as the group sub-codewords. i.i.d. Gaussian signals with $N=3 \times 10^{4}$ signal samples are used to emulate the host signal.

Interleaving collusion and averaging collusion are applied to all the three systems - ECC based fingerprinting, GRACE fingerprinting and the appending group ECC fingerprinting. We examine the probability of successfully detecting one colluder $\left(P_{d}\right)$ at WNR (Watermark-to-Noise Ratio) as 0dB in the following three scenarios:

1) Colluders come from a small number of groups. In this case, the grouping correctly reflects the collusion pattern that all the colluders come from a small number of groups. In our simulation, all colluders are from 2 out of 32 groups, and they are randomly distributed between these two groups. The results of $P_{d}$ under interleaving collusion and averaging collusion are shown in Fig. 2 (a) and (b) respectively. When the systems suffer interleaving collusion, we can see that for the same number of colluders, e.g. $c=8$, the $P_{d}$ 's for the proposed GRACE and the appending group ECC fingerprinting are similar and have $10 \%$ improvement over ECC based fingerprinting; when c gets larger, the improvement in $P_{d}$ can be as large as $70 \%$. From another point of view, if we require the $P_{d}$ of the system to be no less than a certain value e.g. $98 \%$, the number of colluders the system can resist can be improved from 6 colluders (for ECC based fingerprinting) to 18 colluders (for the proposed GRACE fingerprinting). Under the averaging collusion, all systems have $P_{d}$ close to 1 , but we still can see $2 \%$ improvement on $P_{d}$ brought by GRACE fingerprinting.

2) Colluders randomly distribute across all groups. In this case, the grouping does not capture the collusion pattern. The colluders randomly distributed across all groups. The results under interleaving and averaging collusion are shown in Fig. 2 (c) and (d) respectively. Under interleaving collusion the proposed GRACE fingerprinting has up to $30 \%$ improvement over ECC based fingerprinting on $P_{d}$ while the appending group ECC fingerprinting performs a little worse than ECC fingerprinting. Under averaging collusion, the proposed GRACE fingerprinting has comparable performance with ECC based fingerprinting.

3) Colluders come from distinct groups. In this case, the grouping knowledge is extremely inaccurate. All the colluders come from distinct groups (i.e. the number of groups equals the number of colluders $c$ ). The results under interleaving and averaging collusion are shown in Fig. 2 (e) and (f) respectively. Even in this case, under interleaving collusion, the proposed GRACE fingerprinting still has up to $20 \%$ improvement over ECC based fingerprinting on $P_{d}$. The appending group ECC fingerprinting performs worse than the ECC based 


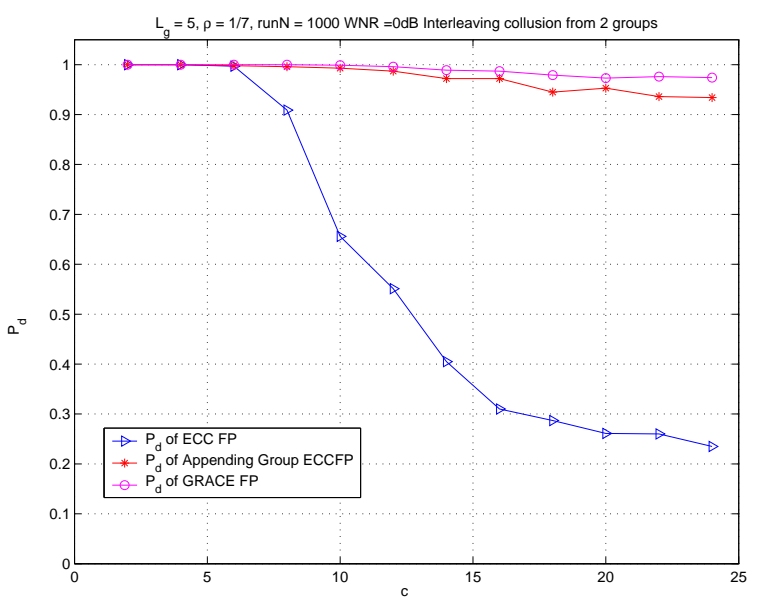

(a)

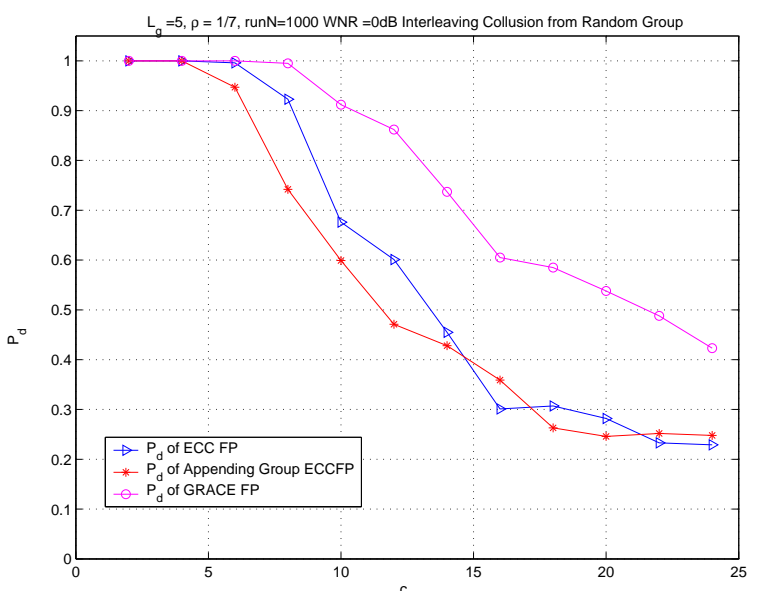

(c)

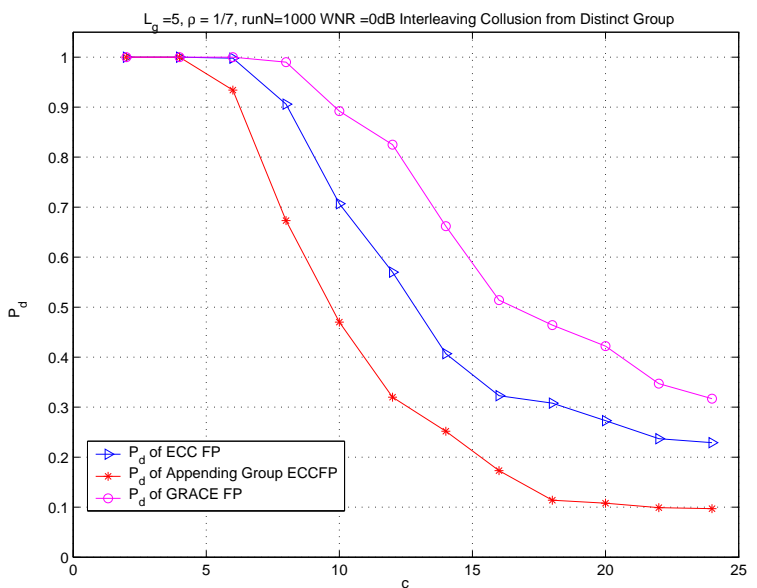

(e)

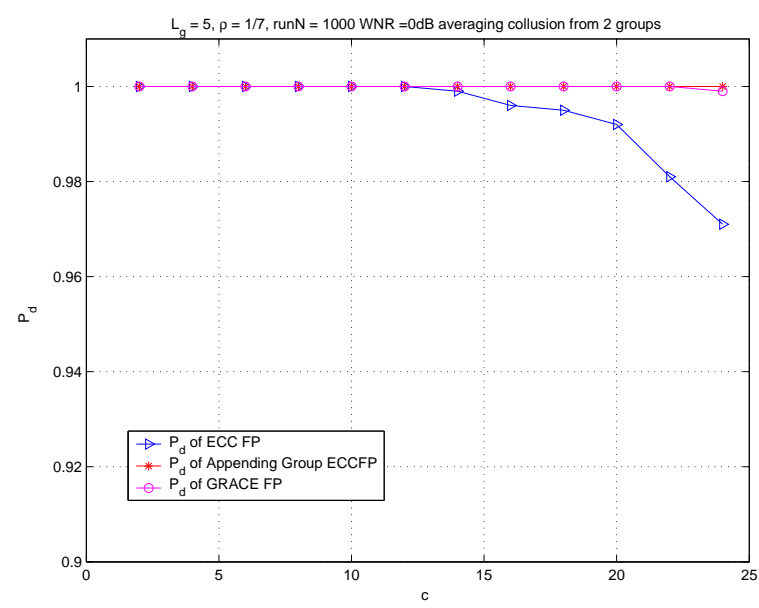

(b)

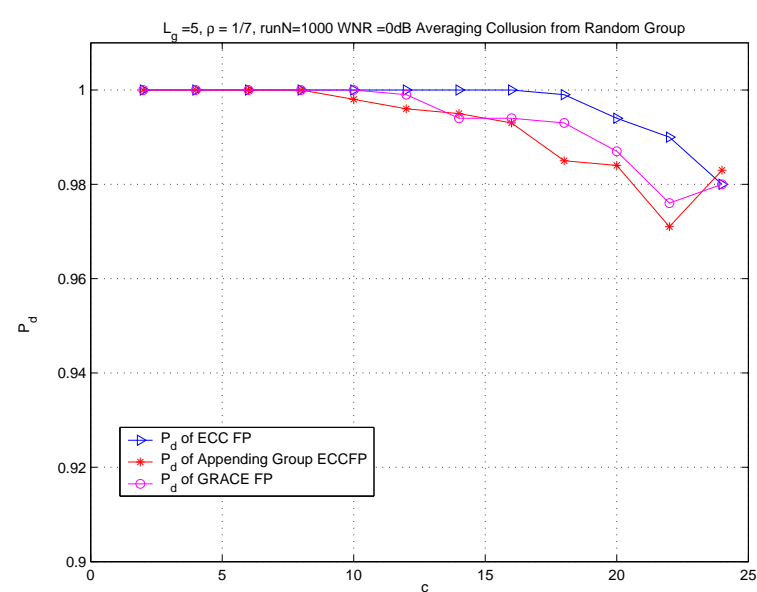

(d)

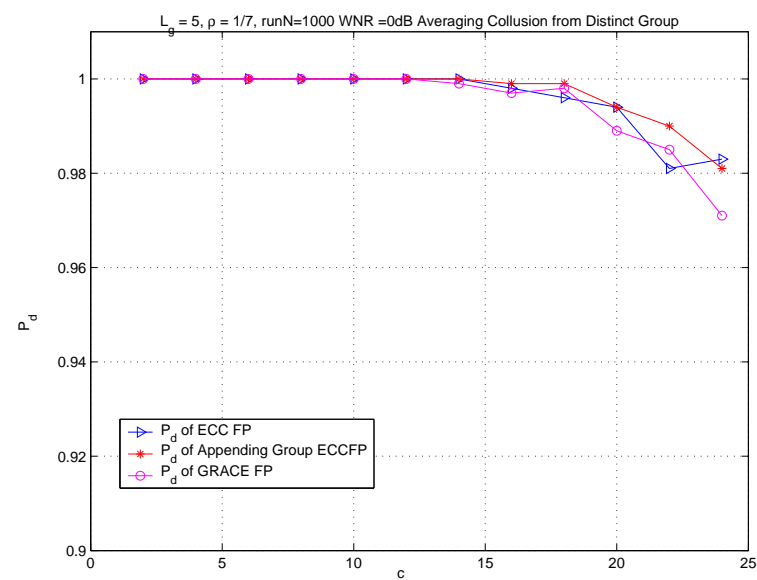

(f)

Figure 2. Performance comparison of the proposed GRACE fingerprinting, appending group ECC fingerprinting and the existing ECC fingerprinting schemes: Probability of detection $P_{d}$ versus the colluder number $c$ at WNR $=0 \mathrm{~dB}$. Colluders come from 2 groups under (a) interleaving collusion and (b) averaging collusion; Colluders are randomly distributed under (c) interleaving collusion and (d) averaging collusion; Colluders come from distinct groups under (e) interleaving collusion and (f) averaging collusion 
fingerprinting by about $15 \%$. Under averaging collusion, the proposed GRACE fingerprinting has comparable performance with ECC based fingerprinting.

The above results can be explained as follows. When collusion happens inside a smaller number of groups, the group information is well preserved so that the group detection for both GRACE and the appending group ECC fingerprinting is of high accuracy. Inside the small number of groups, where the user codewords can be well distinguished due to higher minimum distance, the colluder detection is better than that of non-grouped case. When colluders come from multiple groups or even distinct groups and apply interleaving collusion, for GRACE fingerprinting, the energy of group codeword is reduced after collusion but preserved. Therefore we still have the improvement in detection though it is not as good as the first case. For appending group ECC fingerprinting, when the number of groups gets larger, especially larger than $L_{g}$, under interleaving attack the detector may lose the information of some guilty groups, which results in no performance improvement over ECC based fingerprinting. Under averaging collusion, both group-based schemes have the same group information from the colluders, and their performances are similar. When multiple groups participate the collusion as in the latter two cases, the energy of the group information is reduced and extracted with low accuracy, leading to the diminishing performance gain over ECC based fingerprinting.

The comparison between the GRACE fingerprinting and the appending group ECC fingerprinting demonstrates the significant performance improvement obtained by the joint consideration of coding and embedding. Apart from the collusion resistance gain, we shall analyze the advantage of the joint coding and embedding over the appending group ECC fingerprinting in terms of security in the next section. We also examined the cases of low WNR. The proposed GRACE has substantial improvement over exiting ECC fingerprinting scheme under interleaving collusion for the case that grouping accurately reflects the collusion pattern. For the other two scenarios it has comparable performance with ECC fingerprinting. Overall, the joint coding and embedding along with the grouping brings a consistent improvement of the proposed GRACE fingerprinting over the existing ECC based fingerprinting under various scenarios.

\subsection{Experimental Results with Real Images}

In order to demonstrate the effectiveness of the proposed joint coding and embedding techniques, we apply the proposed approach to real images and compare its collusion resistance performance with that of the existing ECC fingerprinting. We use the additive spread spectrum watermarking scheme for fingerprint embedding, where the original image is divided into $8 \times 8$ blocks and the fingerprint is added into the block DCT coefficients after perceptually weighting. The fingerprint basis is generated according to i.i.d. Gaussian distribution $N(0,1)$. In this experiment, the original host signal is assumed to be available and we have non-blind detection.

We select $512 \times 512$ Lena and Baboon as original images to demonstrate the performance of the proposed fingerprinting system on images with different natures. We apply both the existing ECC based fingerprinting and our proposed GRACE fingerprinting on images, examining their performance under interleaving collusion attacks. With the same fingerprint code setup as in Sec.4.1, the effective host signal length is 65670 for Lena and 142200 for Baboon. The fingerprinted images have an average PSNR of $41.7 \mathrm{~dB}$ for Lena and $39.0 \mathrm{~dB}$ for Baboon. Fig. 3 shows the original and fingerprinted images along with the corresponding difference images. The results of 500 iterations at $\mathrm{WNR}=0 \mathrm{~dB}$ on real images against collusion inside 2 groups are shown in Fig. 4 . We can get similar conclusions to what we get from synthetic signals.

\section{DISCUSSIONS}

\subsection{Security of the Group Information}

From the proposed scheme we can see that the group information helps to narrow down the suspicious users in the colluder detection. However, if the group information is not embedded properly, the attackers may figure out the group sub-codeword positions and frame innocent groups to mislead the detection. Therefore, the group information should have sufficiently high security. In the following, we shall examine the security of the group information for GRACE Fingerprinting and compare it with that of the appending group ECC fingerprinting.

For the appending group ECC fingerprinting, all the users inside one group have the same group sub-codeword with length $L_{g}$, thus they have $L_{g}$ segments in common. While for users coming from different groups, their 

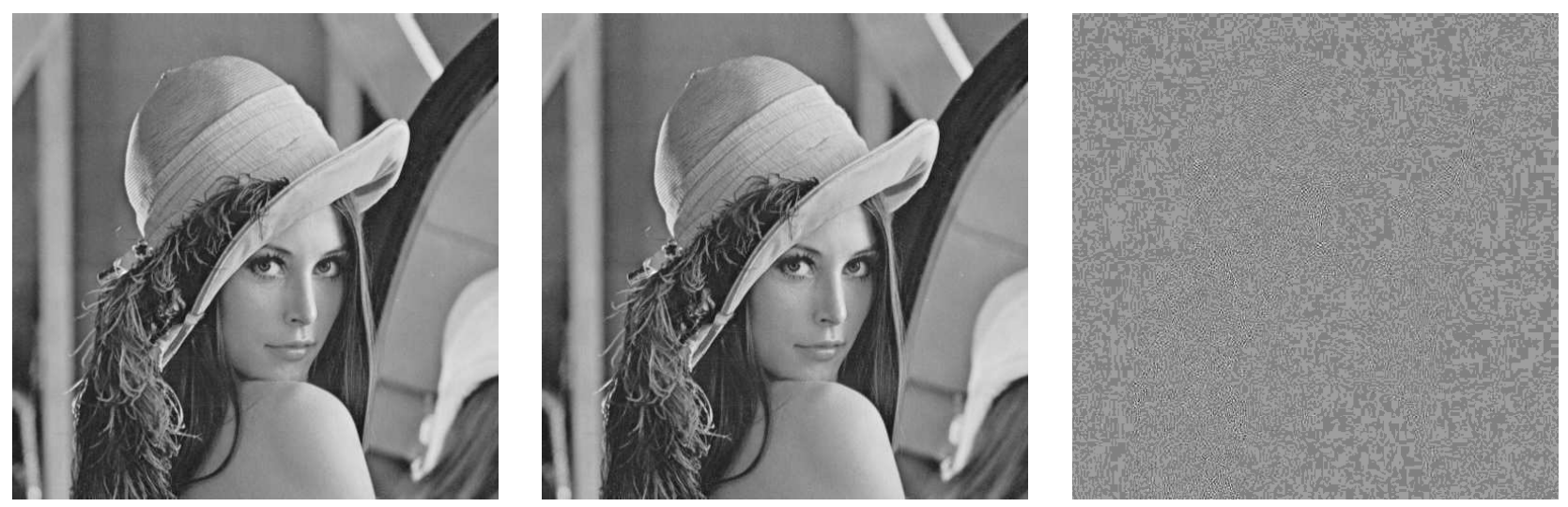

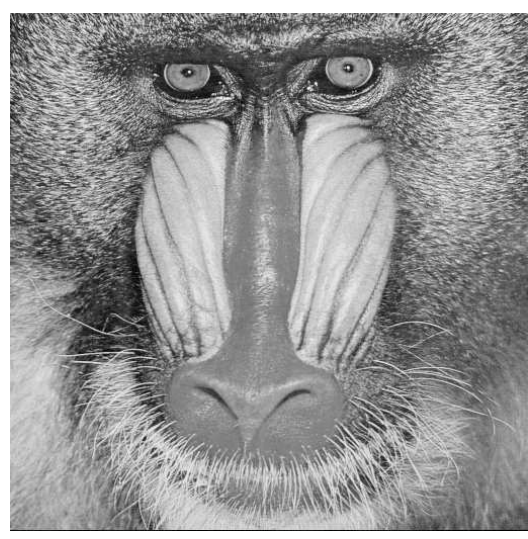

(a)

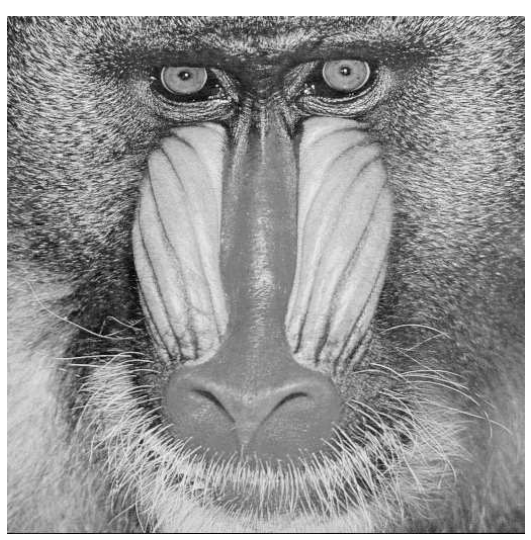

(b)

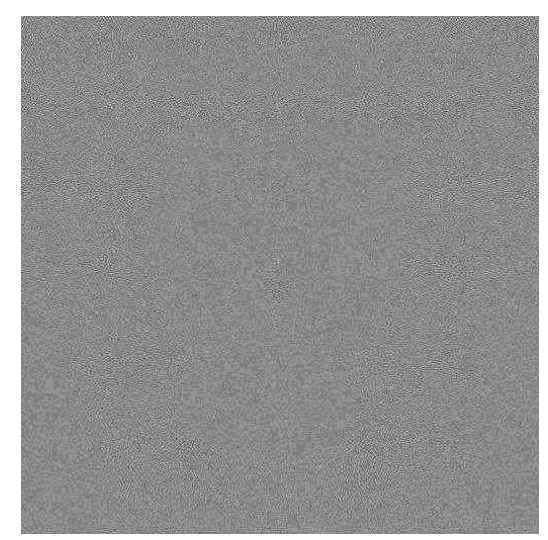

(c)

Figure 3. (a) Original Host Images (b) Fingerprinted Images (c) Corresponding Difference Images

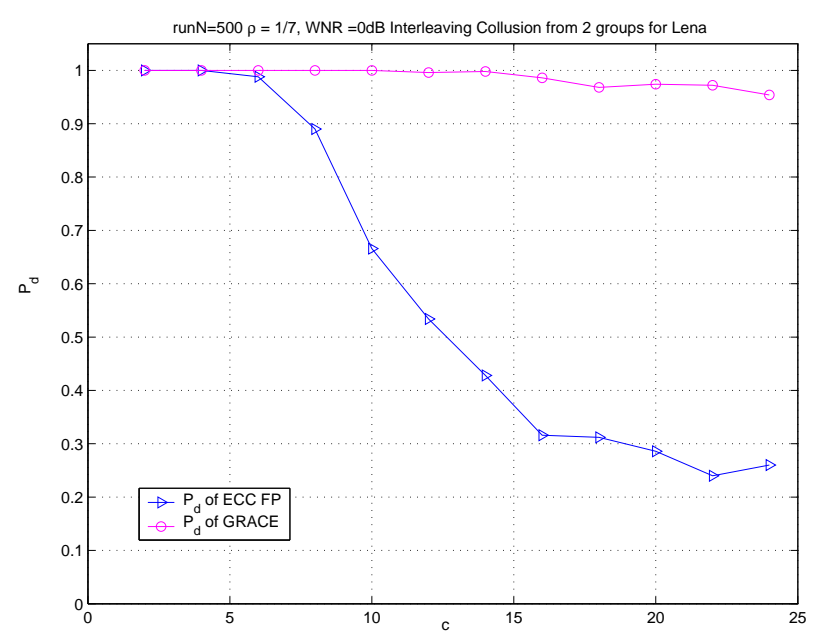

(a)

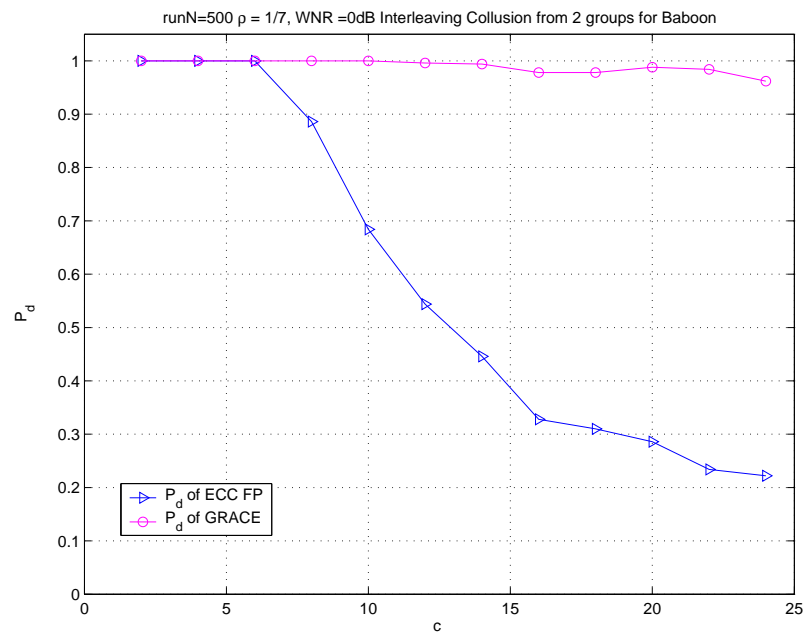

(b)

Figure 4. Experimental results for real image of (a) Lena and (b) Baboon under interleaving collusion by colluders from 2 groups. 
matches in the user sub-codewords are at most $L-D$ which is usually much smaller than $L_{g}$. When several users compare their copies, by examining the number of matched segments they can figure out whether they belong to one group or not. They can even identify the group sub-codeword positions. With the information of the group sub-codeword positions, one colluder may contribute his/her share only to group sub-codeword positions and another colluder from a different group only contributes to user sub-codeword positions. Under this attack, after the group detection, the colluder detection will be limited in the group that only one colluder comes from. Since this colluder did not contribute the user sub-codeword, he/she is less likely to be declared as the colluder. Hence the probability of accusing an innocent user will be very high.

For GRACE fingerprinting, each group has different group sub-codeword from the others. Within one group users have different user sub-codewords. As a result of overlapping these two sub-codewords, the fingerprint sequence for each user is different from any other user. Therefore colluders cannot figure out the grouping information by comparing their copies. We further note that immaterial of the segment the colluder contributes, he/she always contributes both the group information and user information. The attack described above cannot succeed here. Combined with the collusion resistance results in Section 4, the joint coding and embedding of GRACE provides a more secure and effective way to incorporate the group information.

\subsection{Computational Complexity of GRACE Fingerprinting Detection}

The detection of the proposed GRACE fingerprinting consists of two main stages: the group detection and the colluder detection. The computational complexity of the group detection is $O(g N)$ from the correlation detector, where $g$ is the total number of groups and is usually much less than the user number $N_{u}$. In the colluder detection, the demodulation step correlates test segments with the orthogonal sequence for each symbol to determine the extracted codeword. Since the fingerprint sequences for each segment only have $q$ different versions (corresponding to $q$ symbols), we need $O(q N)$ computational complexity for this step. The decoding step of the colluder detection needs at most $L N_{u}$ comparisons. Since normally $L N_{u}<q N$ and $g$ is at the order of $q$, the total computational complexity of the proposed system is $O(q N)$. When Reed-Solomon code is employed, the detection computational complexity becomes $O\left(\sqrt[t]{N_{u}} N\right)$ as $N_{u}=q^{t}$. This is basically the same as the complexity of the conventional ECC based fingerprinting and remains considerably lower than $O\left(N_{u} N\right)$ complexity of the orthogonal fingerprinting.

It is worth mentioning that since in most cases the colluder detection is applied within a small amount of groups, the suspicious user set will be much smaller than that in non-grouped ECC based fingerprinting. This helps to bring a even faster detection process during the colluder detection stage.

\subsection{Multi-level GRACE Fingerprinting}

The idea of the proposed GRACE fingerprinting is to use the group information to narrow down the suspicious colluders to a smaller group. Within each group the minimum distance between the users' codewords are larger than that of the whole user set so that the users' codewords are more separated. Following this idea, we can extend our GRACE fingerprinting to a general multi-level GRACE fingerprinting to capture a more complicated collusion pattern.

We partition (or build) the codebook with minimum distance $D_{0}$ into groups. Inside each group the minimum distance $D_{1}$ is larger than $D_{0}$. Then we repeat this partition for each group until the minimum distance equals the code length or the structure of the group can capture the collusion pattern. When combining the group information with the user information, we adopt the same strategy used in the tree-based scheme in [3] to assign each level an orthogonal sequence and embed them by proper scaling. At the detector side, the group information at each level is used one by one to narrow down the suspicious colluders. Then the colluder can be detected inside the extracted groups as before.

\section{CONCLUSIONS}

Because of cultural and other social reasons, users often form a collusion group in a foreseeable pattern. Taking advantage of this observation and based on our previous study, we have proposed a group-based joint coding and embedding fingerprinting system. In this system, the fingerprint for each user is compact and consists of user 
sub-codeword and group sub-codeword, which are embedded overlappingly in host signal via spread spectrum technique. The detection is done in two levels, which identifies guilty groups through correlation and then narrows down to specific colluders through minimum distance decoding inside the extracted guilty groups. Simulation results show that the proposed fingerprinting system can provide substantial improvement over existing ECC based fingerprinting. The group information helps to limit the suspicious users to a smaller set, and leads to a higher probability of detection.

\section{REFERENCES}

1. M. Wu, W. Trappe, Z. Wang, and K.J.R. Liu, "Collusion Resistant Fingerprinting for Multimedia", IEEE Signal Processing Magazine,pp 15-27, March, 2004.

2. Z.J. Wang, M. Wu, H. Zhao, W. Trappe, and K.J.R. Liu, "Resistance of Orthogonal Gaussian Fingerprints to Collusion Attacks," Proc. of ICASSP, pp 724-727, Apr. 2003.

3. Z.J. Wang, M. Wu, W. Trappe, and K.J.R. Liu, "Group-Oriented Fingerprinting for Multimedia Forensics," EURASIP Journal on Applied Signal Processing (special issue on Multimedia Security and Rights Management), 14, Nov. 2004.

4. D. Boneh and J. Shaw, "Collusion-secure Fingerprinting for Digital Data," IEEE Tran. on Info. Theory, 44(5), pp. 1897-1905, 1998.

5. A. Barg, G.R. Blakley and G. Kabatiansky "Digital fingerprinting codes: Problem statements, constructions, identification of traitors" IEEE Trans. Inform. Theory, 49(4), pp. 852-865, April 2003.

6. G. Tardos, "Optimal probabilistic fingerprint codes" Proceedings of the 35th Annual ACM Symposium on Theory of Computing, 2003.

7. D. To, R. Safavi-Naini and Y. Wang, "A 2-secure code with efficient tracing algorithm", Progress in Cryptology - INDOCRYPT'02, Lecture Notes in Computer Science, Vol. 2551, Springer-Verlag, pp. 149-162, 2002.

8. J.N. Staddon, D. R. Stinson, and R. Wei, "Combinatorial Properties of Frameproof and Traceability Codes", IEEE Trans. on Information Theory, vol. 47, no. 3, March 2001.

9. R. Safavi-Naini and Y. Wang, "Collusion Secure q-ary Fingerprinting for Perceptual Content," Security and Privacy in Digital Rights Management (SPDRM'01), pp. 57-75, 2002.

10. M. Fernandez, and M. Soriano, "Soft-Decision Tracing in Fingerprinted Multimedia Content", IEEE Multimedia, Vol.11 No.2, pp. 38-46, April-June 2004.

11. S. He and M. Wu, "Performance Study of ECC-based Collusion-resistant Multimedia Fingerprinting", in Proceedings of the 38th CISS, pp. 827-832, March 2004.

12. I. Cox, J. Killian, F. Leighton, and T. Shamoon, "Secure Spread Spectrum Watermarking for Multimeida", IEEE Transactions on Image Processing, Vol.6, No.12, pp. 1673-1687, Dec 1997.

13. M. Wu and B. Liu, "Data Hiding in Image and Video: Part-I - Fundamental Issues and Solutions", IEEE Trans. on Image Proc., vol.12, no.6, pp.685-695, June 2003. 